\title{
Special SeCtion Guest EDITORIAL
}

\section{Photonics in the Auditory System}

We are extremely delighted to present a collection of seven papers in this special section of the Journal of Biomedical Optics that reports on applications of photonics in studying the auditory system. While optical methods have been used by a variety of investigators to study a wide range of biological systems, the utility of such methods in probing the auditory system represents an emerging area in biophotonics.

The collection covers a broad range, representing the breadth and the diverse potential applications of photonics. The first three papers are directed toward understanding some of the physical characteristics of the inner and outer hair cells (OHCs), the latter being regarded as the mechanical amplifiers within the cochlea. In the first paper, Greeson and Raphael present a theoretical model for fluorescence polarization consistent with the cylindrical shape of the OHCs, and experimental implementation of fluorescence polarization microscopy to study OHC plasma membrane properties. The second paper, by Organ and Raphael, reports on fluorescence recovery after photobleaching as a method to characterize the mobility of prestin, a transmembrane protein that is believed to be unique to the $\mathrm{OHCs}$ and involved in $\mathrm{OHC}$ electromotility. In the third paper, Tiede et al. report on utilization of twophoton fluorescence microscopy to assess the metabolic state of both inner and outer hair cells, and relate the measurements to different mitochondrial distributions in these cell types.

The next two papers study the organ of Corti. Jacob et al. describe a confocal imaging system coupled with Fourier analysis to measure sound-induced motion of the organ of Corti. Chen et al. report an optical coherence tomography system to acquire in vivo images and vibration measurements of the organ of Corti of the guinea pig.
Finally, the last two papers are related to the effects of laser irradiation on the auditory system. Wenzel et al. report on collagen remodeling and new collagen deposition within the basilar membrane of the organ of Corti of mice in response to pulsed laser irradiation at $694 \mathrm{~nm}$ in conjunction with dye staining of the basilar membrane. Izzo et al. describe spatially selective activation of gerbil auditory neurons using pulsed laser irradiation at $2.12 \mu \mathrm{m}$.

We hope that this collection of papers will be of interest to the biophotonics community in general, and the inner ear specialists in particular. While this collection may be the first compilation of scholarly articles dedicated to the application of photonics in the auditory system, it is our hope that this may just be the beginning of a new age for probing hearing with light.

Bahman Anvari, Ph.D. University of California, Riverside, USA

William E. Brownell, Ph.D. Baylor College of Medicine, Houston, USA

Dennis M. Freeman, Ph.D. Massachusetts Institute of Technology, Cambridge, USA

Mats Ulfendahl, Ph.D. Karolinska Institutet, Stockholm, Sweden

Special Section Guest Editors 